\title{
Conditioned food preferences
}

\author{
ANTHONY SCLAFANI \\ Brooklyn College and The Graduate School, City University of New York, Brooklyn, New York
}

\begin{abstract}
Animals learn to prefer the flavors of foods on the basis of the foods' postingestive nutritional consequences. This has been demonstrated with the conditioned flavor preference paradigm. With this paradigm, one flavor (the $\mathrm{CS}+$ ) is paired with a nutrient that is orally consumed or is infused via a post-oral (e.g., intragastric) route; another flavor (CS-) is paired with a nonnutritive source. In subsequent two-choice tests, the rats displayed reliable preferences for the $\mathrm{CS}+$ flavor over the CS - flavor. Very strong preferences (>95\%) for sour or bitter flavors have been conditioned in nondeprived rats by pairing the $\mathrm{CS}+$ with intragastric infusions of Polycose during 24-h/day training sessions. These conditioned preferences persisted for several weeks when the CS + flavor was no longer paired with IG nutrient infusions. Thus, with the appropriate training procedures, conditioned flavor preferences can be as robust as conditioned flavor aversions.
\end{abstract}

In deciding which foods to select and reject, omnivores like the rat are guided by both innate and acquired flavor preferences and aversions. Much attention has been focused on the rat's inborn preference for the sweet taste of sugar, although recent work suggests that rats also have innate attractions to the taste of starch and the flavor of fat (Ackroff, Vigorito, \& Sclafani, 1990; Hall \& Bryan, 1981; Vigorito \& Sclafani, 1988). Rats avoid, on the other hand, bitter and sour tastes and show either a preference for or an aversion to salty tastes, depending on the salt concentrations and the rats' sodium repletion/depletion state (Hall \& Bryan, 1981; Nachman, 1962). These inherited predispositions presumably aid the animal in selecting nutritious foods and avoiding toxic or imbalanced foods.

With experience, animals refine their preferences and aversions as they associate the flavors of specific foods with the foods' postingestive consequences. There is extensive evidence that rats, along with many other species, readily learn to avoid the flavor of foods or fluids that produce visceral malaise (Barker, Best, \& Domjan, 1977). Such aversions are formed even with respect to highly preferred sweet substances (e.g., sugar solutions) that are followed by toxicosis (e.g., $\mathrm{LiCl}$ injection), so that the conditioned animal reacts to the "sweet" as if it had an unpalatable bitter taste (Berridge, Grill, \& Norgren, 1981). Less well documented, on the other hand, is the conditioning of food preferences based on postingestive nutritional benefits. In particular, previous attempts to convert flavor aversions to preferences by means of nutritional conditioning have been unsuccessful (Rozin, Gruss, \& Berk, 1979). This has led to the view that flavor pref-

The author gratefully acknowledges the contributions of Karen Ackroff, Graciela Elizalde, François Lucas, Jeffrey W. Nissenbaum, and Catalina Perez to the research described in this paper. This research was supported by grants from the National Institute of Diabetes and Digestive and Kidney Diseases (DK31135) and the Research Foundation of the City University of New York. Reprint requests should be sent to Anthony Sclafani, Department of Psychology, Brooklyn College, Brooklyn, NY 11210. erence conditioning is inherently more difficult than aversion conditioning (Rozin \& Zellner, 1985; Zahorik, 1979). However, recent findings from several different laboratories demonstrate that rats can acquire nutrient-based flavor preferences (see, e.g., Bolles, Hayward, \& Crandall, 1981; Booth, 1985; Capaldi, Campbell, Sheffer, \& Bradford, 1987; Holman, 1968; Puerto, Deutsch, Molina, \& Roll, 1976; Sclafani, 1990; Simbayi, Boakes, \& Burton, 1986). The present paper is a brief review of work from my laboratory which shows that with the appropriate training procedures very strong preferences can be conditioned even for normally avoided bitter and sour tastes (see also Sclafani, 1990).

\section{CONDITIONED FLAVOR PREFERENCE PARADIGM}

The standard procedure used to study acquired food preferences is the conditioned flavor preference paradigm (CFP). With this procedure, an arbitrary flavor (the conditioned stimulus, or $\mathrm{CS}+$ ) is paired with a nutrient (the unconditioned stimulus, or US), and a different flavor (the CS -) is paired with a nonnutritive source (e.g., water) or nothing. Following some number of training trials, the animal's preference for the CS + and $C S-$ flavors is assessed in two-bottle choice tests. There are many variations on this paradigm. For example, the nutrient US may be delivered via an oral route (i.e., the animal consumes the nutrient by mouth) or by a post-oral route (e.g., the animal is given an intragastric nutrient infusion).

\section{ORAL CONDITIONING STUDIES}

With respect to the oral route, it has been reported in several studies that rats will acquire preferences for a CS + flavor mixed into a nutrient solution or powdered food; this is a simultaneous conditioning procedure, since the $\mathrm{CS}+$ and US are presented together. Conditioned flavor preferences have been obtained with various nutrient USs, 
including sugars, hydrolyzed starch (e.g., Polycose), starch, fat, protein, and ethanol (Bolles et al., 1981; Elizalde \& Sclafani, 1988; Mehiel \& Bolles, 1988). For example, we (Elizalde \& Sclafani, 1988) recently trained rats to drink a CS + flavored Polycose solution (the US) and CS - flavored water on separate days; the CSs were saccharin-sweetened cherry or grape flavors. In subsequent two-choice tests with flavored water, the rats displayed a $90 \%$ preference for the CS + flavor over the CS - flavor. Following a second training session, however, this preference weakened to $70 \%$, apparently because the animals learned to discriminate between the CS + flavored Polycose and CS + flavored water.

Animals have also been trained according to a trace conditioning procedure, in which they consume the CS + (flavored water) and then, following a delay, the nutrient US. In general, the trace procedure has been less effective than the simultaneous procedure in conditioning flavor preferences, but significant $C S+$ preferences have been obtained with delays as long as $1 \mathrm{~h}$ between CS + and US presentation (Capaldi et al., 1987; Elizalde \& Sclafani, 1988; Holman, 1975; Simbayi et al., 1986). The ability of animals to learn CS + preferences with the delayed presentation of the nutrient US is of interest, because in the normal process of ingestion and digestion there is some delay between the orosensory effects and the postingestive consequences of food.

A potential complication with oral conditioning procedures is that the flavor of the nutrient may facilitate or interfere with conditioning of the CS + flavor preference. Facilitation may occur through the process of flavor-flavor conditioning. That is, animals may learn to prefer the CS + flavor because they associate it with the flavor of the nutrient (e.g., the sweet taste of sugar) rather thanor in addition to-the postingestive consequences of the nutrient. Flavor-flavor conditioning has been formally demonstrated in experiments in which a cue flavor was paired with a palatable but nonnutritive saccharin solution or mineral oil emulsion (Elizalde \& Sclafani, 1990; Holman, 1975). Although flavor-flavor conditioning is an interesting phenomenon in its own right (Rozin \& Zellner, 1985), it is a potential confound in the analysis of the postingestive conditioning effects of nutrients (referred to here as flavor-nutrient conditioning).

One approach taken to minimize the impact of flavorflavor associations in food preference conditioning is to use "bland" tasting nutrients such as starch (Bolles et al., 1981; Capaldi et al., 1987; Simbayi et al., 1986). However, recent findings indicate that rats are very attracted to the flavor of starch and starch-derived polysaccharides, so that data obtained with these carbohydrates cannot be taken as unambiguous evidence for flavor-nutrient conditioning (Sclafani, 1987). Mehiel and Bolles (1988) evaluated the contribution of nutrient flavor in food conditioning by comparing the effects obtained with nutrient USs that differed in their initial palatability (sucrose, Polycose, corn oil, ethanol). They obtained comparable CS + preferences with the different nutrients and therefore con- cluded that caloric value rather than flavor of the nutrient USs was the primary reinforcer of the CS + flavor preference (but see Elizalde \& Sclafani, 1990).

Another way to control for flavor-flavor conditioning is to add the nutrient US to the CS + and the CS - flavors and to manipulate the postingestive effects of the US using a gastric fistula. That is, the rat is trained to eat the CS + flavored US solution with a closed gastric fistula ("real feeding'"), so that nutrient is normally digested and absorbed, and the CS - flavored US solution with an open gastric fistula ("sham feeding"), so that the nutrient leaves the digestive system unabsorbed. In the first study of this type, Van Vort and Smith (1983) failed to obtain a conditioned preference for a CS + flavored milk diet that was real-fed over a CS - flavored diet that was sham-fed. However, in a more recent experiment, we observed a reliable CS + preference in rats real- and sham-fed flavored Polycose solutions (Nissenbaum, Sclafani, Vigorito, \& Cassouto, 1988; Sclafani, 1990).

As noted above, the flavor of the nutrient may interfere with rather than facilitate preference conditioning in some situations; the nutrient flavor may overshadow the $\mathrm{CS}+$ flavor, for example. This is particularly likely in the trace conditioning procedure, in which the animal consumes the CS + and then, after a delay, the US. In this case, the flavor of the nutrient is temporally more closely associated with the nutrient's postingestive actions than is the CS + flavor. One way to minimize the overshadowing effect of the nutrient flavor is to use a backward conditioning procedure-that is, to present the CS + flavor after the US. Boakes and Lubart (1988) reported that rats learned to prefer a CS + flavor that was consumed 3 min after the intake of a glucose US solution. A different tactic used by Elizalde and Sclafani (1988) was first to train animals that the flavor of the nutrient was not associated with postingestive reinforcement. This was accomplished through the preexposure of one group of rats to a Polycose solution containing acarbose, a drug that inhibits carbohydrate digestion; a second group was preexposed to Polycose without acarbose. When subsequently trained to associate a CS + flavor with the delayed (10$\mathrm{min}$ ) presentation of a Polycose solution, the rats in the Polycose-acarbose group acquired a stronger CS + preference than did the rats in the Polycose-no-drug group.

\section{POST-ORAL CONDITIONING}

To eliminate the flavor of the nutrient as a potential confound in food conditioning studies, one can deliver the nutrient by a route that bypasses the oral cavity. Significant flavor preferences have been obtained with intragastric infusions of complete diets or of glucose, casein, oil, or ethanol and intravenous or hepatic-portal infusions of glucose (Baker, Booth, Duggan, \& Gibson, 1987; Holman, 1968; Mather, Nicolaidis, \& Booth, 1978; Puerto et al., 1976; Sherman, Hickis, Rice, Rusiniak, \& Garcia, 1983; Tordoff \& Friedman, 1986). However, preferences have not been obtained in all experiments, and in some 
cases, flavor aversions were conditioned through nutrient infusions (Deutsch, Molina, \& Puerto, 1976). These negative results, however, should not detract from the positive findings, since the post-oral administration of nutrients can be problematic. First, the experimenter-determined infusions may be excessive in size, rate, and/or concentration, and as a result, they may produce visceral discomfort (Booth, 1985). Second, nutrients delivered by non-oral routes may not be normally processed by the gastrointestinal system because of the absence of salivary enzymes (see Deutsch, 1990).

In most previous nutrient conditioning studies, fooddeprived animals have been trained in short, daily sessions (but see Mather et al., 1978). Recent work in my laboratory indicates that training nondeprived rats in long (24-h/day) sessions is very effective in conditioning flavor preferences (Elizalde \& Sclafani, 1990; Lucas \& Sclafani, 1989; Sclafani \& Nissenbaum, 1988). The rats in our studies were fitted with two chronic intragastric (IG) catheters that were connected through a swivel device to two infusion pumps. The pumps were automatically operated whenever the rats drank from one or two drinking tubes containing flavored or unflavored water; the intragastric infusion rate approximated the oral ingestion rate. With this system, the animals were free to eat chow and drink fluid normally, and they controlled the number and size of IG infusions they received each day.

In two initial studies (Elizalde \& Sclafani, 1990; Sclafani \& Nissenbaum, 1988), rats were trained by giving them ad-lib access on 1 day to CS + flavored water, paired with IG infusions of Polycose (16\% or $32 \%)$. On the next day, the rats were given access to CS - flavored water, paired with IG water infusions. The cue flavors were unsweetened grape or cherry Kool Aid, and the CS + and CS - flavors were counterbalanced across subjects. In addition, the right-left positions of the flavors were counterbalanced across days. After 4 training days, flavor preferences were assessed by giving the rats access to both the $\mathrm{CS}+$ and $\mathrm{CS}-$ for 2 days paired with their appropriate infusions.

After one or two training cycles, the rats in both studies displayed very strong preferences ( $>90 \%$ ) for the CS + over the CS - (Elizalde \& Sclafani, 1990; Sclafani \& Nissenbaum, 1988). The rats also preferred the CS+ to plain water $(\approx 90 \%)$, but they showed no reliable preference between the CS - flavor and plain water. The latter findings indicate that the animals had developed a true preference for the CS + rather than an aversion for the CS-. Since intake of the CS + was paired with IG Polycose infusions in the two-choice tests, it could be argued that the rats selected the CS + in order to obtain the IG Polycose, not because they preferred the flavor per se. Subsequent extinction tests revealed that this was not the case; the rats strongly preferred (>90\%) the CS + when both the CS + and the CS - were paired with IG water infusions during 4- or 14-day two-choice tests (Elizalde \& Sclafani, 1990; Sclafani \& Nissenbaum, 1988). Furthermore, the rats continued to reliably prefer the CS + flavor, although at a reduced level ( $\approx 75 \%)$, after they had been returned to their home cages and were given 40 days of two-bottle access to CS + and CS - without IG infusions (Elizalde \& Sclafani, 1990).

We have also conditioned flavor preferences by pairing a grape or cherry flavor with IG infusions of a corn oil emulsion (Lucas \& Sclafani, 1989). The preferences conditioned by the corn oil, however, developed less readily and were weaker than those conditioned by Polycose. Corn oil may be less effective than Polycose in conditioning flavor preferences, because fats are digested and absorbed more slowly than carbohydrates are. Alternatively, there may be qualitative differences in the postingestive signals generated by these nutrients. In either case, these findings indicate that the caloric value of the US nutrient infusion is not the only determinant of flavor preference conditioning.

In our most recent study, rats were conditioned as described above with IG infusions of $32 \%$ Polycose as the US, but the CS stimuli were bitter and sour flavors (Elizalde, 1990). That is, for half the rats (SOA subgroup), .03\% sucrose octa acetate (SOA) was the CS+ paired with IG Polycose, and $.05 \%$ citric acid (CA) was the CS - paired with IG water; for the remaining rats (CA subgroup), the CS flavors were reversed. Additional rats that served as controls were exposed to the same training procedures, except that both flavors were paired with IG water infusions. As indicated in Figure 1, the data from the control group show that the SOA and CA flavors were, as intended, equally acceptable in the one-bottle training trials and equally preferred in the two-bottle tests. Furthermore, the bitter and sour flavors were equally unpreferred in two-bottle tests with plain water.

To illustrate that the bitter and sour flavors were equally effective as CS + stimuli, the data from the SOA and CA subgroups are presented separately in Figure 1. Following the one-bottle conditioning trials, the rats in both subgroups displayed near-total preferences $(96 \%-98 \%)$ for the CS + over the CS - in the two-bottle tests. Furthermore, the SOA group showed a $94 \%$ preference for its bitter CS + over plain water, and the CA group showed a $97 \%$ preference for its sour CS + over plain water. Both groups, however, strongly preferred $(\approx 95 \%)$ plain water to their CS - flavor.

The near-total preference displayed by the experimental rats for their CS+ flavors over plain water is in marked contrast to the $\approx 70 \%$ preference that the control rats showed for plain water over the bitter and sour flavors. Thus, pairing the bitter or sour taste with IG Polycose converted a flavor aversion to a very strong learned preference. Note, on the other hand, that the experimental rats displayed a stronger aversion than did the control rats to the sour and bitter tastes when they were the CS stimuli. This may represent a negative contrast effectthat is, switching the experimental rats from the strongly preferred CS + to the CS - may have elicited an enhanced aversive response to the $\mathrm{CS}$ - flavor.

Another new finding that emerged from this study is that the IG Polycose conditioned an increased acceptance (i.e., increased absolute intake) of the CS+ flavor. That 

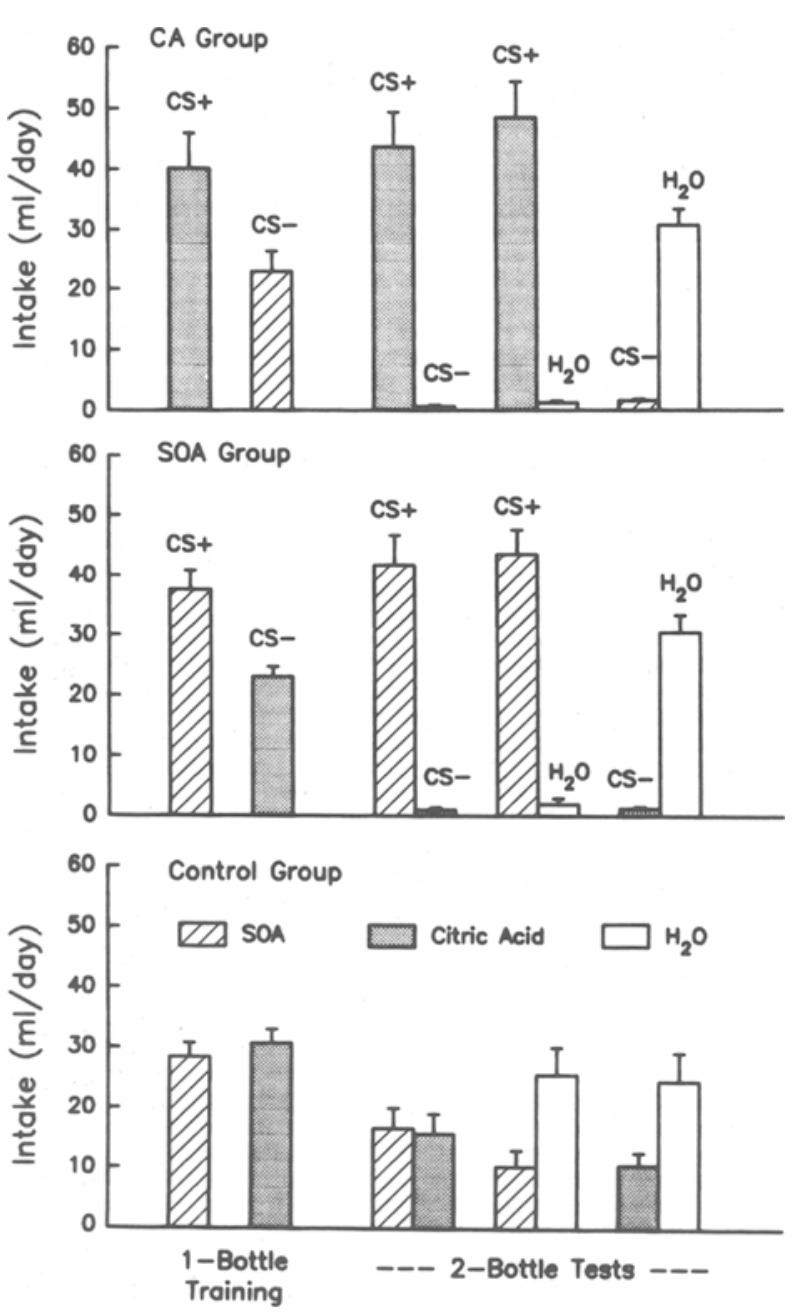

Figure 1. Mean $(+S E)$ oral intakes of sucrose octa acetate solution (SOA), citric acid solution (CA), and plain water $\left(\mathrm{H}_{2} \mathrm{O}\right)$. The one-bottle data represent the intakes from the second of two 4-day training sessions; the two-bottle data represent the intakes from 2-day two-bottle tests that followed the second training session. The $\mathrm{CA}$ group $(n=8)$ had citric acid $(C S+)$ paired with IG Polycose infusions and SOA (CS -) paired with IG water infusions, whereas the SOA group $(n=8)$ had SOA $(C S+)$ paired with IG Polycose and citric acid (CS-) paired with IG water infusions. The control group $(n=8)$ had both citric acid and SOA paired with IG water infusions; water intake in all groups was paired with IG water infusions. Data from Elizalde (1990).

is, as is illustrated in Figure 1, the experimental rats consumed $\approx 30 \%$ more of the sour and bitter CS + solutions than did the control group during the one-bottle training trials. The experimental subgroups' one-bottle intake of the CS + also exceeded their intake of plain water in the water versus $\mathrm{CS}-$ test (Figure 1). In subsequent onebottle acceptance tests conducted in the home cage with no IG infusions, the experimental rats also consumed more $\mathrm{CS}+$ than plain water during short (3.6 vs. $1.2 \mathrm{ml} / 30$ $\mathrm{min}$ ) and long (60 vs. $43 \mathrm{ml} / 24-\mathrm{h}$ ) sessions (Elizalde, 1990). In our previous conditioning studies, we did not observe increased acceptance of cherry- or grape-flavored $\mathrm{CS}+$ solution; also the CS + preference developed less rapidly with the cherry/grape flavors than with the bitter/ sour flavors (Elizalde \& Sclafani, 1990). These differences in conditionability may have occurred because the grape/cherry flavors are less distinctive to the animals than the bitter/sour flavors are; note that citric acid is a common ingredient in the grape and cherry Kool Aid mixtures. Thus, flavor preference conditioning may be influenced by the specific CSs as well as USs used (see also Elizalde \& Sclafani, 1988).

The strong preference and increased acceptance for the $\mathrm{CS}+$ flavors suggests that the experimental rats came to "like" the bitter and sour tastes. Rozin and Zellner (1985) have argued, however, than an increased flavor preference does not necessarily indicate a change in the hedonic evaluation of the flavor. That is, animals (including humans) may come to prefer a flavor because they expect a beneficial consequence (e.g., recovery from illness) rather than because they come to "like" the flavor. There are no firmly established criteria to distinguish between hedonic conditioning and expectancy learning, although Rozin and Zellner (1985) suggest that preferences based on liking are more stable across physiological states and environmental contexts and are longer lasting than preferences based on expected outcomes. By these criteria, the CS + preferences conditioned by IG Polycose would appear to involve an enhanced hedonic evaluation of the $\mathrm{CS}+$ flavor, since these preferences are long-lasting and are displayed in both test cages and home cages as well as under both nondeprived and food-deprived conditions (Ackroff \& Sclafani, unpublished observations; Elizalde, 1990; Elizalde \& Sclafani, 1990).

Another criterion used to evaluate hedonic conditioning involves orofacial expressive responses (Rozin \& Zellner, 1985). Because rats display distinctive patterns of ingestive and aversive orofacial reactions to intraoral infusions of taste stimuli such as sucrose and quinine (Grill \& Berridge, 1985), these orofacial reactions have been taken as measures of positive and negative hedonic tone, although this interpretation is not universally accepted (Booth, 1990). Elizalde (1990) recently investigated the effects of IG Polycose conditioning on the orofacial reactions to a sour or bitter CS + flavor. As described above, the rats displayed a strong preference and increased acceptance for the CS + . Nevertheless, they did not show increased ingestive reactions to the CS + flavor, relative to the CS flavor, during 1-min intraoral infusion tests. This finding, in contrast to the other data cited above, suggests that the preference conditioned by IG Polycose was not associated with an increased liking for the CS + flavor. This issue requires further study; it is possible that the orofacial test employed in our laboratory was not sufficiently sensitive to reveal a change in the hedonic evaluation of the bitter and sour CS+ flavors.

\section{SUMMARY}

It is now firmly established that the postingestive nutritional effects of food can condition strong flavor preferences in rats. Furthermore, not only can preferences be 
acquired for neutral flavors, but also, with the appropriate procedures, aversions to bitter and sour tastes can be converted into strong preferences. Conditioned flavor preferences may also be associated with an increased acceptance of the cue flavor and may be remarkably resistant to extinction. These results suggest that nutrient conditioning produces a long-lasting enhancement in the palatability of the CS + flavor, but the conditioning effects on flavor hedonics remain to be clarified. Finally, the view that animals are inherently less capable of learning flavor preferences as opposed to flavor aversions is no longer tenable in light of the recent preference conditioning studies. Although it is indeed the case that flavor aversions are more readily produced in the laboratory than flavor preferences are, this is due to the fact that it is easier to inject a toxic drug than to administer a physiologically appropriate nutrient infusion to an animal.

\section{REFERENCES}

ACKroff, K., Vigorito, M., \& SClafanı, A. (1990). Fat appetite in rats: The response of infant and adult rats to nutritive and non-nutritive oil emulsions. Appetite, 15, 171-188.

Baker, B. J., Booth, D. A., Duggan, J. P., \& Gibson, E. L. (1987). Protein appetite demonstrated: Learned specificity of protein-cue preference to protein need in adult rats. Nutrition Research, 7, 481-487.

Barker, L. M., Best, M. R., \& Domjan, M. (Eds.) (1977). Learning mechanisms in food selection. Waco, TX: Baylor University Press.

BerRIDGe, K. C., GRILl, H. J., \& Norgren, R. (1981). Relation of consummatory responses and preabsorptive insulin release to palatability and learned taste aversions. Journal of Comparative \& Physiological Psychology, 95, 363-382.

BOAKES, R. A., \& LUBART, T. (1988). Enhanced preference for a flavour following reversed flavour glucose pairing. Quarterly Joumal of Experimental Psychology, 40, 49-62.

Bolles, R. C., Hayward, L., \& Crandall, C. (1981). Conditioned taste preferences based on caloric density. Journal of Experimental Psychology: Animal Behavior Processes, 7, 59-69.

Booth, D. A. (1985). Food-conditioned eating preferences and aversions with interoceptive elements: Conditioned appetites and satieties. Annals of the New York Academy of Sciences, 443, 22-41.

Boorh, D. A. (1990). How not to think about immediate dietary and postingestional influences on appetites and satieties. Appetite, 14, 171-179.

Capaldi, E. D., Campbell, D. H., Sheffer, J. D., Bradford, J. P. (1987). Conditioned flavor preferences based on delayed caloric consequences. Joumal of Experimental Psychology: Animal Behavior Processes, 13, 150-155.

DEuTSCH, J. A. (1990). Food intake: Gastric factors. In E. M. Stricker (Ed.), Neurobiology of food and fluid intake (pp. 151-182). New York: Plenum.

Deutsch, J. A., Molina, F., \& Puerto, A. (1976). Conditioned taste aversion caused by palatable nontoxic nutrients. Behavioral Biology, 16, 161-174.

ElizALDE, G. (1990). Conditioned carbohydrate appetite in rats. Unpublished doctoral dissertation, City University of New York.

EluzalDE, G., \& SCLAFANI, A. (1988). Starch-based conditioned flavor preferences in rats: Influence of taste, calories, and CS-US delay. Appetite, 11, 179-200.

Eluzalde, G., \& Sclafani, A. (1990). Flavor preferences conditioned by intragastric Polycose infusions: A detailed analysis using an electronic esophagus preparation. Physiology \& Behavior, 47, 63-77.

GriLl, H. J., BeRrIDGE, K. C. (1985). Taste reactivity as a measure of the neural control of palatability. In J. M. Sprague \& A. N. Epstein (Eds.), Progress in psychobiology and physiological psychology (pp. 161). New York: Academic Press.

HALL, W. G., \& Bryan, T. E. (1981). The ontogeny of feeding in rats: IV. Taste development as measured by intake and behavioral responses to oral infusions of sucrose and quinine. Journal of Comparative \& Physiological Psychology, 95, 240-251.

Holman, G. L. (1968). Intragastric reinforcement effect. Joumal of Comparative \& Physiological Psychology, 69, 432-441.

HolmaN, E. W. (1975). Immediate and delayed reinforcers for flavor preferences in the rat. Learning \& Motivation, 6, 91-100.

LuCAS, F., SClafani, A. (1989). Flavor preferences conditioned by intragastric fat infusions in rats. Physiology \& Behavior, 46, 403-412.

Mather, P., Nicolaidis, S., Booth, D. A. (1979). Compensatory and conditioned feeding responses to scheduled glucose infusions in the rat. Nature, 273, 461-463.

MeHIEL, R., Bolles, R. C. (1988). Learned flavor preferences based on calories are independent of initial hedonic value. Animal Learning \& Behavior, 16, 383-387.

NACHMaN, M. (1962). Taste preferences for sodium salts by adrenalectomized rats. Journal of Comparative \& Physiological Psychology, 55, 1124-1129.

Nissenbaum, J. W., Sclafani, A., Vigorito, M., \& Cassouto, K. (1988). Conditioned flavor preferences in sham-feeding rats. Proceedings of the Eastern Psychological Association Meeting, 59, 18.

Puerto, A., Deutsch, J. A., Molina, F., Roll, P. (1976). Rapid rewarding effects of intragastric injections. Behavioral Biology, 18, 123-134.

Rozin, P., Gruss, L., Berk, G. (1979). Reversal of innate aversions: Attempts to induce a preference for chili peppers in rats. Journal of Comparative \& Physiological Psychology, 93, 1001-1014.

Rozin, P., \& Zellner, D. (1985). The role of Pavlovian conditioning in the acquisition of food likes and dislikes. Annals of the New York Academy of Sciences, 443, 189-202.

SCLAFANI, A. (1987). Carbohydrate taste, appetite, and obesity: An overview. Neuroscience \& Biobehavioral Reviews, 11, 131-153.

SCLAFANI, A. (1990). Nutritionally-based learned flavor preferences in rats. In E. D. Capaldi \& T. L. Powley (Eds.), Taste, experience, and feeding (pp. 139-156). Washington, DC: American Psychological Association.

SClafani, A., \& Nissenbaum, J. W. (1988). Robust conditioned flavor preference produced by intragastric starch infusions in rats. American Journal of Physiology, 255, R672-R675.

Sherman, J. E., Hickis, C. F., Rice, A. G., Rusiniak, K. W., \& GARCIA, J. (1983). Preferences and aversions for stimuli paired with ethanol in hungry rats. Animal Learning \& Behavior, 11, 101-106.

Simbayi, L. C., Boakes, R. A., \& Burton, M. J. (1986). Can rats learn to associate a flavour with the delayed delivery of food? Appetite, 7, 41-53.

ToRDOFF, M. G., \& Friedman, M. I. (1986). Hepatic-portal glucose infusions decrease food intake and increase food preference. American Journal of Physiology, 251, R192-R196.

VAN VorT, W., SMITH, G. P. (1983). The relationship between the positive reinforcing and satiating effects of a meal in the rat. Physiology \& Behavior, 30, 279-284.

Vigorito, M., \& Sclafani, A. (1988). Ontogeny of Polycose and sucrose appetite in neonatal rats. Developmental Psychobiology, 21, 457-465.

ZAHORIK, D. M. (1979). Learned changes in preferences for chemical stimuli: Asymmetrical effects of positive and negative consequences, and species differences in learning. In J. H. A. Kroeze (Ed.), Preference behaviour and chemoreception (pp. 233-245). London: Information Retrieval Ltd. 